\title{
等离子喷涂-物理气相沉积的气相沉积机理
}

\author{
邓子谦 ${ }^{1,2}$, 刘 敏 ${ }^{2,3}$, 毛 杰 $^{2}$, 张小锋 ${ }^{2}$, 陈文龙 ${ }^{2}$, 陈志坤 ${ }^{2}$
}

(1. 广东工业大学 材料与能源学院, 广州 510006; 2. 广东省新材料研究所, 现代材料表面工程技术国家工程实验 室, 广东省现代表面工程技术重点实验室, 广州 510651; 3. 中南大学 材料科学与工程学院, 长沙 410083)

摘 要: 以等离子喷涂-物理气相沉积(PS-PVD)喷涂团聚的 $\mathrm{ZrO}_{2}-7 \mathrm{wt} \% \mathrm{Y}_{2} \mathrm{O}_{3}(7 \mathrm{YSZ}$ )粉末在五个喷距下制备了热障 涂层。通过场发射-扫描电镜(FE-SEM)和 X射线衍射(XRD)分析了五个涂层样品的微观结构和相成分差异。另外, 通 过发射光谱(OES)诊断研究了射流中 7YSZ 粉末气相浓度随喷距的变化。最后, 阐述了 3 种不同的气相沉积涂层生 长机制, 说明了射流中粉末的状态和气相浓度对涂层结构的影响。研究表明: (1)350 mm 和 $1800 \mathrm{~mm}$ 喷距下形成的 均是致密结构涂层, 而 650 1250 mm 喷距下形成的是典型的 PS-PVD 柱状结构涂层。(2)350 mm 喷距下制备的涂层 由四方相 $\left(\mathrm{t}^{\prime}\right)$ 和单斜相 $(\mathrm{m})$ 氧化锆构成; 当喷距大于 $650 \mathrm{~mm}$ 时, 涂层以四方相 $\left(\mathrm{t}^{\prime}\right)$ 氧化锆为主。(3)350 $\mathrm{mm}$ 喷距下涂 层是由高浓度气相过饱和自发形核形成的新核和液/固粒子共同作用形成的; 喷距 650 1250 $\mathrm{mm}$ 下, 涂层生长以气 相沉积于基体进行非自发形核为主, 气相在射流中的自发形核为辅; 喷距 $1800 \mathrm{~mm}$ 下涂层由气相过冷凝固的粒子 堆积而成。

关 键 词: PS-PVD; 涂层生长; 自发形核; 非自发形核; 光谱诊断

中图分类号: TQ174 文献标识码: A

\section{Deposition Mechanism Based on Plasma Spray-Physical Vapor Deposition}

\author{
DENG Zi-Qian ${ }^{1,2}$, LIU Min ${ }^{2,3}$, MAO Jie ${ }^{2}$, ZHANG Xiao-Feng ${ }^{2}$, CHEN Wen-Long ${ }^{2}$, CHEN Zhi-Kun²
}

(1. School of Materials and Energy, Guangdong University of Technology, Guangzhou 510006, China; 2. National Engineering Laboratory for Modern Materials Surface Engineering Technology, The Key Lab of Guangdong for Modern Surface Engineering Technology, Guangdong Institute of New Materials, Guangzhou 510651, China; 3. School of Materials Science and Engineering, Central South University, Changsha 410083, China)

\begin{abstract}
Thermal barrier coatings (TBCs) were prepared via plasma spray-physical vapor deposition (PS-PVD) technique at five spray distances by using agglomerated $\mathrm{ZrO}_{2}-7 \mathrm{wt} \% \mathrm{Y}_{2} \mathrm{O}_{3}$ (7YSZ) powders. The microstructure and phase composition of these coatings under different spray distances were analyzed by field emission-scanning electron microscope (FE-SEM) and X-ray diffraction (XRD). Besides, gas concentration of the powders in plasma jet was diagnosed with stand-off distances by Optical Emission Spectroscopy (OES). Eventually, three formation mechanisms of the PS-PVD coatings based on vapor deposition were proposed, and the relationship between the concentration of gas phase and the microstructure of the coatings was explained. The results reveal that: (1) the coatings prepared at spray distance of $350 \mathrm{~mm}$ and $1800 \mathrm{~mm}$ exhibit dense structure, while typical PS-PVD 7YSZ columnar coatings were formed at spraying distance of $650-1250 \mathrm{~mm}$; (2) the coating formed at $350 \mathrm{~mm}$ spraying distance is composed of
\end{abstract}

收稿日期: 2017-02-20; 收到修改稿日期：2017-04-27

基金项目: 广东省自然科学基金团队项目(2016A030312015); 广东省广州市科学技术合作项目(2013B050800027, 201508030001, 2011C007); 广东省科学院项目(2017GDASCX-0843, 2016GDASPT-0206, 2016GDASPT-0317)

Guangdong Natural Science Foundation Project (2016A030312015); Science and Technology Cooperation Project of Guangdong Province, and of Guangzhou City (2013B050800027, 201508030001, 2011C007); Platform Construction Project of Guangdong Academy of Science (2017GDASCX-0843, 2016GDASPT-0206, 2016GDASPT-0317)

作者简介: 邓子谦(1988-), 男, 博士研究生. E-mail: dengziqian0404@163.com

通讯作者: 刘 敏, 教授. E-mail: liumin_gz@163.net 
$\mathrm{t}^{\prime}$ - and $\mathrm{m}-\mathrm{ZrO}_{2}$, and others are mainly composed of $\mathrm{t}^{\prime}-\mathrm{ZrO}_{2}$ at over $650 \mathrm{~mm}$ stand-off distance; (3) At $350 \mathrm{~mm}$ spray distance, the coating is developed under the combination effect of the supersaturated spontaneous nucleation from high concentration of the gas phase and liquid/solid particles. At spraying distance of 650-1250 mm, the growth of columnar coatings are dominated by the non-spontaneous nucleation after the deposition of gas phase on the substrate, and supplemented by spontaneous nucleation in plasma jet. At spraying distance of $1800 \mathrm{~mm}$, the coating is accumulated from spontaneous re-solidified gas phase particles.

Key words: PS-PVD; coating growth; non-/spontaneous nucleation; spectral diagnosis

等离子喷涂-物理气相沉积(Plasma Spray-Physical Vapor Deposition, PS-PVD)是 20 世纪末基于低压等 离子喷涂(Low Prossure Plasma Spray, LPPS)发展出 来的新一代等离子喷涂技术 ${ }^{[1-4]}$ 。相比于传统的 LPPS, PS-PVD 具有更低的工作压力、更高的喷涂功 率和更大的工作气体流量, 从而可以实现气液固多 相沉积 ${ }^{[5-9]}$ 。通过 PS-PVD 气相沉积制备的热障涂层 具有优异的隔热和抗热震性能, 使其成为未来最有 前景的先进发动机热障涂层制备技术之一 ${ }^{[10-16]}$ 。

随着工艺研究的深入, 涂层性能的提升遇到瓶 颈, 依靠单纯的工艺摸索来改变涂层的结构或提升 涂层的性能已非常困难, 因此需要进一步了解 PS-PVD 沉积机理, 依靠理论指导工艺实践, 提高 研究效率, 从而更快推动 PS-PVD 走向产业化应用。 Mauer 等 ${ }^{[17-20]}$ 提出了基于沉积速率与基体温度的 PS-PVD 区域结构模型。Gao 等 ${ }^{[21-23]}$ 研究了不同轴 向和径向涂层结构, 归纳出五种典型的 PS-PVD 涂 层结构, 并解释其形成原因。广东省新材料研究所 的 Zhang 等 ${ }^{[24-25]}$ 研究了通过喷距/基体温度和基体 材料对涂层结构的影响，提出了基于 PS-PVD 均匀 形核和非均匀形核的理论。这些工作都为涂层结构 调控和性能提升提供了重要参考。但是, PS-PVD 沉 积机理探索得还不够完善。Mauer 和 Gao 只是从宏 观角度解释了涂层结构的形成, 而 Zhang 的理论涉 及到微观形核, 但无法解释 PS-PVD 柱状涂层生长的 一些现象，如柱状结构分支。

基于前人的研究结果, 本工作着重结合光谱诊 断和 PS-PVD 喷涂实验, 构建射流中气相粉末浓度 与涂层结构的关系, 从形核角度探讨 PS-PVD 轴向 气相沉积机制。

\section{1 实验方法}

\section{1 样品制备及涂层表征}

以镍基高温合金 K417(14-16Co, 8.5-9.5Cr, 4.85.7 Al, 4.5-5.0 Ti, 2.5-3.5Mo, 0.13-0.22C, rest $\mathrm{Ni}$ )为基 体材料, 切割成 $\phi 25.4 \mathrm{~mm} \times 7 \mathrm{~mm}$ 圆柱状试样。首先 对其表面进行打磨、超声波除油与抛光处理; 然后,
采用 PS-PVD(Multicoat ${ }^{\mathrm{TM}}$ PS-PVD system, OerlikonMetco)工艺在抛光基体上沉积陶瓷层。喷涂所用粉 末是球形团聚的 $7 \mathrm{YSZ}$ 粉末 $\left(\mathrm{ZrO}_{2}-7 \mathrm{wt} \% \mathrm{Y}_{2} \mathrm{O}_{3}\right.$, 粒径 5 22 $\mu \mathrm{m}$, Mecto 6700, Oerlikon-Metco), 如图 1 所 示。喷涂陶瓷层的参数如表 1 所示。

涂层制备完成后, 采用场发射扫描电镜(FESEM, Nova-Nono430, FEI)观察涂层微观结构; 采用 XRD (Smartlab, Rigaku)分析涂层的相成分。

\section{2 光谱诊断}

涂层的结构差异与粉末气相在射流中的分布与 浓度、基体温度等息息相关。当忽略基体影响时, 涂 层的结构主要与气相沉积在基体前的浓度和状态有 关 ${ }^{[20]}$ 。

通过采用光谱仪(Optical Emission Spectroscopy, OES)诊断不同喷距下射流中心的 $\mathrm{Zr}$ 峰强度, 可以 定性表征粉末气相浓度随喷距的变化。PS-PVD 射 流光谱诊断系统布置和详细的诊断方法参见文献 [26]。使用 IHR550 (HORIBA, Japan) 光谱仪, 选用 的光栅型号为 530-27-141, 测量范围为 400 1400 nm,

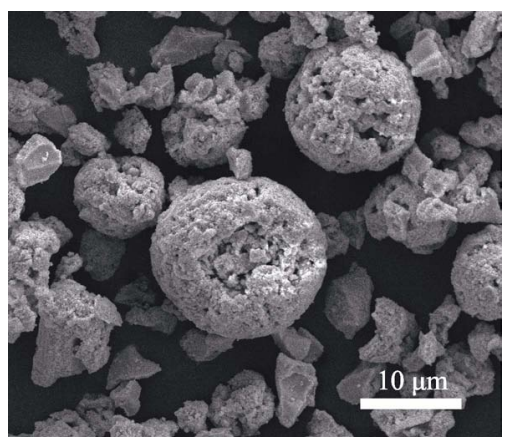

图 1 Mecto 6700 粉末形貌

Fig. 1 Morphology of mecto 6700 powders

表 1 PS-PVD 喷涂 7YSZ 热障涂层参数 Table 1 Parameters of 7YSZ coating by PS-PVD

\begin{tabular}{ccccccc}
\hline Gun & $\begin{array}{c}\text { Power/ } \\
\mathrm{kW}\end{array}$ & $\begin{array}{c}\mathrm{Ar} / \\
\text { slpm }\end{array}$ & $\begin{array}{c}\mathrm{He} / \mathrm{slpm} \\
\text { pressure/Pa }\end{array}$ & $\begin{array}{c}\text { Chamber } \\
\text { preed rate/ } \\
\left(\mathrm{g} \cdot \mathrm{min}^{-1}\right)\end{array}$ & $\begin{array}{c}\text { Stand-off } \\
\text { distance/ mm }\end{array}$ \\
\hline O3CP & 127 & 35 & 60 & 150 & 20 & $\begin{array}{c}350,650,950, \\
1250,1800\end{array}$ \\
\hline
\end{tabular}


狭缝宽度为 $0.02 \mathrm{~mm}$, 曝光时间为 $0.3 \mathrm{~s}$ 。实验结果采 取六次测量取平均值的方法, 以减少周围光照强度 变化引起的误差。

\section{2 结果和讨论}

\section{1 涂层的微观结构分析}

图 2 是 650、950、1250 mm 喷距下样品的截面 和表面形貌。从图 2 中可以看出, 3 个喷距的涂层均 为典型的柱状结构特征, 说明涂层沉积以气相为 主 $^{[12]}$ 。比较 3 个喷距下制备涂层的截面和表面, 可 以发现 3 个涂层的结构仍存在较明显的差异。随机 选取涂层表面一定数目的花菜头结构（10 15 个)， 计算其平均尺寸, 研究发现 $650 \mathrm{~mm} 、 950 \mathrm{~mm}$ 、 $1250 \mathrm{~mm}$ 喷距下涂层表面花菜头的平均尺寸依次为 38、49、62 $\mu \mathrm{m}$ （误差 $\pm 20 \%$ ）。此外，喷距 $650 \mathrm{~mm}$ 和 $950 \mathrm{~mm}$ 表面比较平整, 而 $1250 \mathrm{~mm}$ 表面起伏比 较大, 它们的粗鋉度依次为 3.8、4.3、6.3 $\mu \mathrm{m}$ 。这 些差异与涂层沉积过程中的柱状结构二次枝晶生长 相关。随着喷距增大, 涂层中的单个柱状结构的上 层枝晶数量越多, 涂层表面的花菜头尺寸也相应增 加, 最终造成涂层表面粗粮度也增大。

图 3 是喷距为 350 和 $1800 \mathrm{~mm}$ 制备的涂层截面 和表面形貌, 从图中可以看出, 350 和 $1800 \mathrm{~mm}$ 喷距 涂层均为致密涂层, 前者更致密一些。此外, 两者还
存在明显差别。350 mm 喷距涂层可观察到晶粒长大 的特征和熔融液滴的存在，而 $1800 \mathrm{~mm}$ 喷距涂层则 完全由大小不一的颗粒堆积而成。

\section{2 涂层的物相分析}

图 4 是初始粉末与五个不同喷距样品的 XRD 图谱，从图中可以看出，原始粉末由大量的单斜相 $\left(\mathrm{m}-\mathrm{ZrO}_{2}\right)$ 和少量的四方相 $\left(\mathrm{t}^{\prime}-\mathrm{ZrO}_{2}\right)$ 和 $\mathrm{Y}_{2} \mathrm{O}_{3}$ 相构成。 粉末通过等离子射流加热, $\mathrm{Y}_{2} \mathrm{O}_{3}$ 相会融入 $\mathrm{ZrO}_{2}$ 相中, 单斜相 $\left(\mathrm{m}-\mathrm{ZrO}_{2}\right)$ 会在 $950^{\circ} \mathrm{C}$ 时转变为四方氧化锆相 $\left(\mathrm{t}-\mathrm{ZrO}{ }_{2}\right)$, 在 $2370^{\circ} \mathrm{C}$ 时再转变为立方相 $\left(\mathrm{c}-\mathrm{ZrO}_{2}\right)$ 。当 温度下降时, 立方相 $\left(\mathrm{c}-\mathrm{ZrO}_{2}\right)$ 先转化为四方氧化锆 相 $\left(\mathrm{t}-\mathrm{ZrO}_{2}\right)$, 微米尺度 $\mathrm{t}-\mathrm{ZrO}_{2}$ 相在室温较难存在, 而 代之以亚稳相 $\mathrm{t}^{\prime}-\mathrm{ZrO}_{2}$ 存在, 所以气化较充分的粉 末沉积的涂层多以 $\mathrm{t}^{\prime}-\mathrm{ZrO}_{2}$ 相存在 ${ }^{[27-28]}$ 。四个喷距 650/950/1250/1800 mm 涂层的 XRD 图谱十分相近, 基本都由 $\mathrm{t}^{\prime}-\mathrm{ZrO}_{2}$ 构成，表明 650 1800 mm 喷距涂 层均由气化较充分的粉末沉积而成。而 $350 \mathrm{~mm}$ 喷 距涂层不仅有 $\mathrm{t}^{\prime}-\mathrm{ZrO}_{2}$ 相, 还存在 $\mathrm{m}-\mathrm{ZrO}_{2}$, 结合图 3(a), 侧面证明了喷距 $350 \mathrm{~mm}$ 下粉末气化不充分, 存在较多的未熔或微熔粒子。

\section{3 光谱诊断(OES)}

图 5 是 OES 测量的不同喷距下 $\mathrm{Zr}$ 峰强度, $\mathrm{Zr}$ 峰强度与所在位置粉末气相浓度及射流温度密切相 关 $^{[17]}$ 。从图 5 可以看出, $\mathrm{Zr}$ 强度在 $350 \mathrm{~mm}$ 处最高, 这是由于 PS-PVD工艺喷枪口及其附近能量最集中,
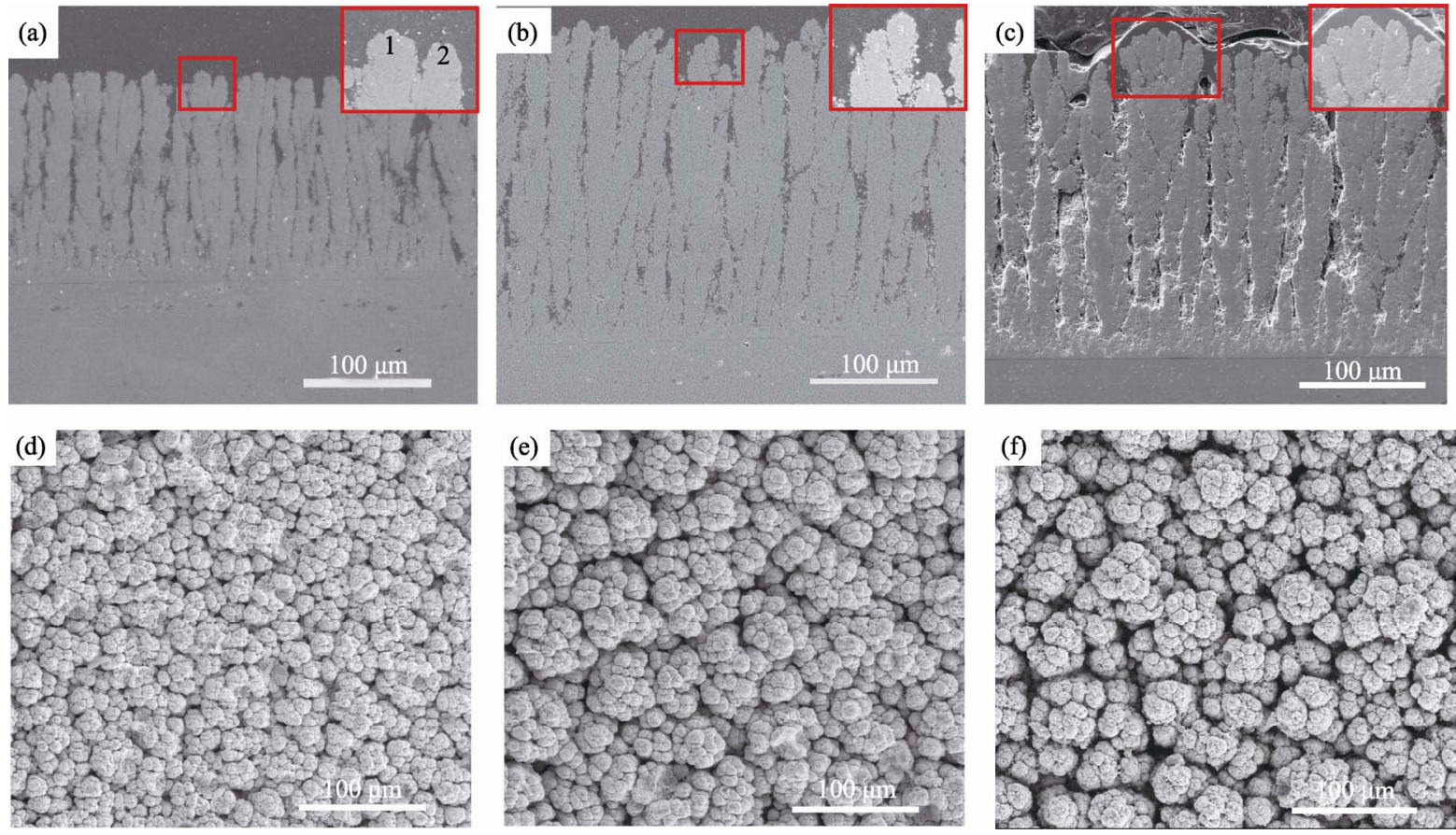

图 2 不同喷涂距离下的 PS-PVD YSZ 柱状结构涂层的 SEM 照片

Fig. 2 SEM morphologies of 7YSZ columnar coating formed at different stand-off distances (a) (d) $650 \mathrm{~mm}$; (b) (e) $950 \mathrm{~mm}$; (c) (f) $1250 \mathrm{~mm}$ 

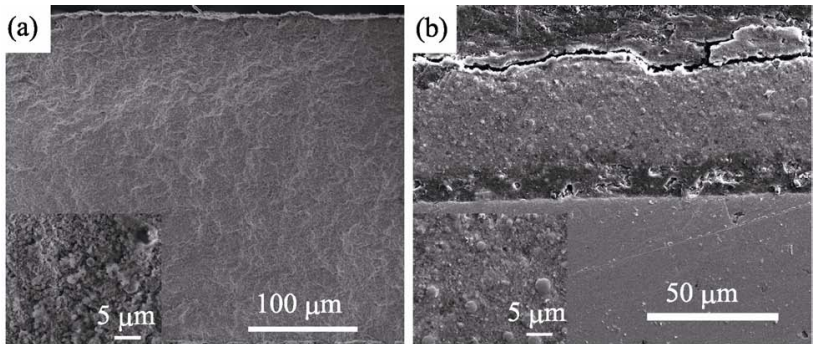

(c)

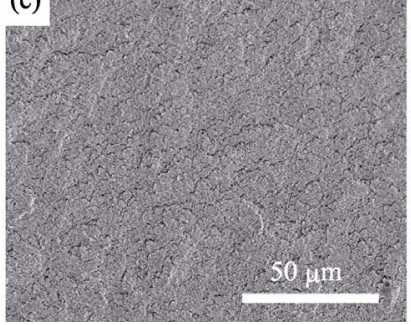

(d)

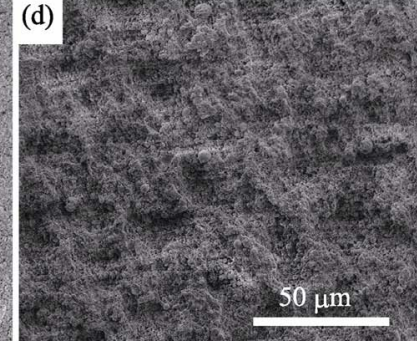

图 3 不同喷涂距离下的 PS-PVD YSZ 致密结构涂层的 SEM 照片

Fig. 3 SEM morphologies of 7YSZ dense coating formed at different stand-off distances

(a) (c) $350 \mathrm{~mm}$; (b) (d) $1800 \mathrm{~mm}$

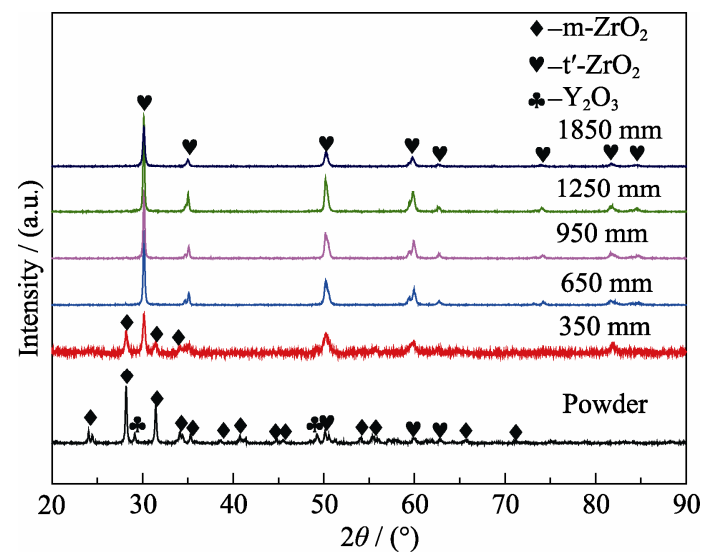

图 4 原始粉末与 5 个喷距样品的 XRD 图谱

Fig. 4 XRD patterns of original powder and the coatings with 5 stand-off distances

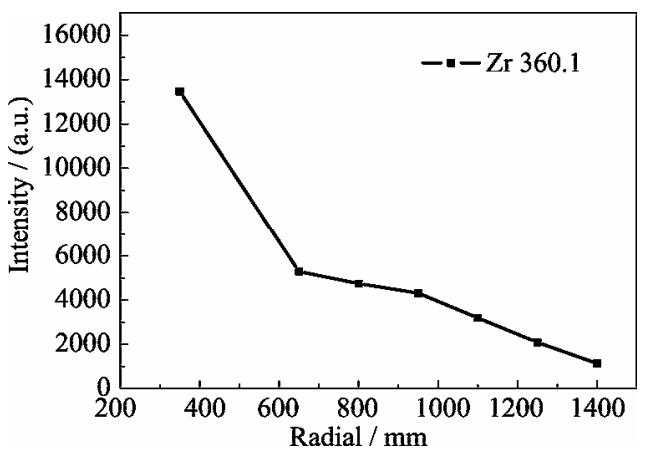

图 5 不同轴向上的光谱诊断 $(\mathrm{Zr}$ 波长 $360.1 \mathrm{~nm})$

Fig. 5 OES diagnostic in different stand-off distances ( $\mathrm{Zr}$ $360.1 \mathrm{~nm}$ )

射流温度最高, 粉末气相浓度最高。随着喷距的增 大, $\mathrm{Zr}$ 峰强度不断降低, 大致可以分为 3 个阶段:
1)350 650 mm 下降的速度最快；2)在喷距 650 $950 \mathrm{~mm}, \mathrm{Zr}$ 强度下降速度变缓; 3) 950 1400 mm(或 之后), $\mathrm{Zr}$ 强度下降速度增加。阶段一到阶段二, $\mathrm{Zr}$ 峰下降速度降低是由于在射流前端, 射流会发生膨 胀, 射流的温度和气相浓度变化梯度很大。随着喷 距的增加, 射流温度下降的梯度减小。阶段三相比 阶段二, 下降速度增加是由于气相粉末飞出射流的 内焰(内焰尾端大约在喷距 $1000 \mathrm{~mm}$ 处), 射流温度 骤降, 导致射流对粉末的加热能力急剧降低, 当周 围环境不足以维持高浓度气相时, 气相开始凝结, 浓度也不断下降。此外, 气相脱离内焰(高粘性 $\mathrm{He}$ 离子)束缚, 分布更宽广, 这也是 $\mathrm{Zr}$ 强度急剧下降 的原因。当达到喷距 $1400 \mathrm{~mm}$ 后, $\mathrm{Zr}$ 峰强度 $<1000$, 说明射流中气相已经较稀薄。

在实际喷涂过程中，基体的侵入会干扰射流， 从而影响射流中粉末的分布和浓度。高速射流撞击 基体形成湍流，在湍流的影响下，基体前方会形成 边界层(boundary layer) ${ }^{[22,29]}$ (见图 6(a)), 气相在边界 层聚集造成浓度升高, 当粉末浓度达到一定之后会 再次达到动态平衡。一般来说, 经过射流的压缩和 膨胀区之后, 离枪口越远(即喷距越大), 射流速度 是不断下降的, 射流密度也越来越稀薄, 所以射流 撞击基体形成的扰动也越来越小，聚集的气相能力 也就减弱了，因此，喷距越短，基体前方的粉末浓 度更高。

\section{4 不同轴向的气相沉积机制}

结合实验和诊断数据, $350 \mathrm{~mm}$ 是以高浓度气相 和少量的液固相混合沉积而成致密结构涂层; 而 $1800 \mathrm{~mm}$ 则是由于气相再凝固的粒子沉积而成致密 结构涂层; 650 1250 $\mathrm{mm}$ 是典型的以气相沉积为主 的柱状涂层结构, 在保持基体温度、材料和粗䊁度 不变的前提下, 涂层差异与射流中气相粉末的浓度 有关。

(1)350 mm 喷距涂层是以高浓度气相和少量的 液固相混合沉积而成致密结构涂层。

先忽略固液两相的影响, 只考虑气相对涂层结 构的影响。喷距 $350 \mathrm{~mm}$ 下, 粉末气相浓度本来就 高, 再加上高速射流撞击基体形成边界层, 造成气 相在基体前方聚集，使得粉末气相浓度进一步提高， 出现局部过饱和, 过饱和的气相由于边界层内湍流 扰动造成的气相浓度、射流温度的起伏从而自发形 核，见图 6。

假设气相自发形核, 形成的晶核为球形, 半径 为 $r$, 会造成系统的体积自由能 $\Delta G_{\mathrm{V}}$ 减少和表面自 由能 $\Delta G_{\mathrm{S}}$ 增加, 系统总的自由能变化满足式(1) ${ }^{[30]}$ : 


$$
\Delta G=\Delta G_{\mathrm{V}}+\Delta G_{\mathrm{S}}=\frac{4}{3} \pi r^{3} \Delta g_{\mathrm{v}}+4 \pi r^{2} \sigma_{\mathrm{vn}}
$$

其中, $\Delta g_{\mathrm{v}}$ 是气相凝结成固相引起的单位体积自由 能量的变化, $\Delta g_{\mathrm{v}}<0 ; \sigma_{\mathrm{vn}}$ 是单位面积的固-气相 界面能。

上式对 $r$ 求偏导并令 $\frac{\partial \Delta G}{\partial r}=0$ 得:

$$
\begin{gathered}
r^{*}=\frac{2 \sigma_{\mathrm{vn}}}{\Delta g_{\mathrm{v}}} \\
\Delta G^{*}=\frac{16 \pi \sigma_{\mathrm{vn}}}{3 \Delta g_{\mathrm{v}}}
\end{gathered}
$$

其中, $r^{*}$ 是临界形核半径; $\Delta G^{*}$ 是临界形核功。

此外, 自发形核率

$$
n^{*}=f\left(n_{1}\right) \exp \left(-\frac{\Delta G^{*}}{K T}\right)
$$

其中, $n_{1}$ 边界层的气相浓度, 一般情况下 $n_{1}$ 越高, $f\left(n_{1}\right)$ 越大。

当气相自发形核是由气相过饱和引起时,

$$
\Delta g_{\mathrm{v}}=\frac{\mathrm{k} T_{\mathrm{g}}}{V_{\mathrm{m}}} \ln \frac{p_{0}}{p_{1}}=-\frac{\mathrm{k} T_{\mathrm{g}}}{V_{\mathrm{m}}} \ln \frac{p_{1}}{p_{0}}=-\frac{\mathrm{k} T_{\mathrm{g}}}{V_{\mathrm{m}}} \ln S
$$

其中, $p_{0} 、 p_{1}$ 分别为气相平衡蒸汽压和非平衡蒸汽 压, $\mathrm{k}$ 为气体常数, $T_{\mathrm{g}}$ 为环境温度, $V_{\mathrm{m}}$ 为单个原子 体积, $S=p_{1} / p_{0}$ 是气相的过饱和度。当过饱和度 $S \leqslant 1$ 时, $\Delta g_{\mathrm{v}} \geqslant 0$, 没有晶核出现; 当过饱和度 $S>1$ 时, $\Delta g_{\mathrm{v}}<0$ 会成为晶核形成的驱动力。

将式(5)代入式(2)和(3),

$$
\begin{gathered}
r^{*}=\frac{2 \sigma_{\mathrm{vn}}}{\Delta g_{\mathrm{v}}}=\frac{2 V_{\mathrm{m}} \sigma_{\mathrm{vn}}}{\mathrm{k} T_{\mathrm{g}} \ln S} \\
\Delta G^{*}=\frac{16 \pi \sigma_{\mathrm{vn}}^{2}}{3 \Delta g_{\mathrm{v}}}=\frac{16 \pi V_{\mathrm{m}}^{2} \sigma_{\mathrm{vn}}^{2}}{3\left(\mathrm{k} T_{\mathrm{g}} \ln S\right)^{2}}
\end{gathered}
$$

由公式(5)、(6)和(7)可知，过饱和度越高，射流 温度越高, 形核尺寸越小, 形核功越小, 形核率 越高。

在 $350 \mathrm{~mm}$ 喷距处具有极高的射流温度和过饱 和度，所以大量小尺寸的新核在边界层形成并沉积 于基体上，在较高的基体温度下这些新核相互聚集 并吸附周围的气相快速生长, 最终形成致密结构涂 层，并具有晶粒长大和取向性不好的特征。

此外，在实际涂层沉积过程中，基体离枪口过 近，粉末粒子还未能完全加热气化，以气、固、液混 合状态沉积涂层, 这也是涂层呈现致密结构的原因 之一。
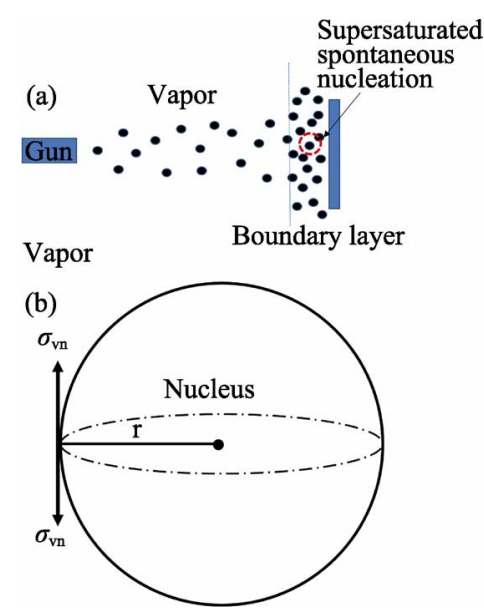

图 6 (a) PS-PVD 的过饱和效应和(b)自发形核

Fig. 6 (a) Saturation effect based on PS-PVD and (b) schematic diagram of spontaneous nucleation

(2)1800 mm 喷距涂层则是由气相再凝固的粒 子堆积而成致密结构涂层。

在 PS-PVD 喷涂过程中, 等离子射流是一个开 放系统, 随着喷涂距离的增加, 等离子射流变得越 来越稀薄, 且射流的温度不断降低 ${ }^{[3]}$, 造成射流对 气相粉末的加热和保温能力不断地减弱, 当气相 7YSZ 所处位置的环境温度低于自身沸点时(接近射 流尾端), 气相 7YSZ 分子、离子或原子将发生过冷 自发形核 ${ }^{[24]}$ 。

考虑过冷度的影响, 故

$$
\Delta g_{\mathrm{v}}=\frac{\Delta H}{T_{\mathrm{m}}} \Delta T
$$

其中, $\Delta H$ 是凝华放热, 故 $\Delta H<0, T_{\mathrm{m}}$ 为材料熔点 温度, $\Delta T$ 是射流环境下的过冷度。

将式(8)代入式(2)和(3), 求得

$$
\begin{gathered}
r^{*}=\frac{2 \sigma_{\mathrm{vn}}}{\Delta g_{\mathrm{v}}}=\frac{2 T_{\mathrm{m}} \sigma_{\mathrm{vn}}}{\Delta H \Delta T} \\
\Delta G^{*}=\frac{16 \pi \sigma_{\mathrm{vn}}^{2}}{3 \Delta g_{\mathrm{v}}}=\frac{16 \pi T_{\mathrm{m}}^{2} \sigma_{\mathrm{vn}}^{2}}{3(\Delta H \Delta T)^{2}}
\end{gathered}
$$

由公式(9)和(10)可知, 过冷度越大, 形核功越 小，临界核越小，(热力学上)形核越容易。但是喷距 的增大，不仅伴随过冷度增大，还有气相浓度 $n_{1}$ 的 降低, 所以, 喷距增加形核率不一定增加(动力学 上), 见式(4)。正常情况下, 当达到一定过冷度后, 气相会先团聚形成晶胚, 再在飞行过程中吸附周围 的气相继续长大，即喷距增大，晶核的尺寸反而越 大，最终沉积于基体上。

(3)650 1250 mm 喷距涂层是典型的以气相沉 积为主的柱状涂层结构。

在 650 1250 mm 阶段, 射流温度适中, 气相浓 
度适中, 只有少量气相粉末在射流中发生过饱和或 过冷自发形核, 大量气相直接吸附与基体后发生非 自发形核, 所以在涂层沉积阶段以非自发形核为主, 涂层初始结构的形成取决于气相与基体的相互作 用 ${ }^{[30-31]}$ 。

当气相 7YSZ 沉积于基体后, 分子、离子或原 子在基体上发生扩散和团聚, 最终非自发形核。图 7 为非自发形核示意图, 在基体 $S$ 上形成球冠晶核 $n$, 半径为 $r$, 接触角为 $\theta$ 。当晶核稳定时,

$$
\sigma_{\mathrm{vs}}=\sigma_{\mathrm{vn}} \cos \theta+\sigma_{\mathrm{ns}}
$$

其中, $\sigma_{\mathrm{vs}} 、 \sigma_{\mathrm{vn}} 、 \sigma_{\mathrm{ns}}$ 分别为气相与基体、气相与 晶核、基体与晶核的表面能。

形成一个晶核总的自由能的变化为:

$$
\begin{aligned}
& \Delta G=\Delta G_{\mathrm{V}}+\Delta G_{\mathrm{S}}= \\
& V \Delta g_{\mathrm{v}}+\sigma_{\mathrm{vn}} S_{1}+\sigma_{\mathrm{ns}} S_{2}-\sigma_{\mathrm{vs}} S_{2}= \\
& \left(\frac{4}{3} \pi r^{3} \Delta g_{\mathrm{v}}+4 \pi r^{2} \sigma_{\mathrm{vn}}\right)\left(\frac{2-3 \cos \theta+\cos ^{3} \theta}{4}\right)
\end{aligned}
$$

其中, $V$ 是冠状晶核的体积; $S_{1}$ 是晶核的上表面(晶 核与气相的接触面)的表面积; $S_{2}$ 是晶核的下表面 (晶核与基体的接触面)的表面积。

上式对 $r$ 求偏导并令 $\frac{\partial \Delta G}{\partial r}=0$ 得:

$$
\begin{gathered}
r^{*}=-\frac{2 \sigma_{\mathrm{vn}}}{\Delta g_{\mathrm{v}}} \\
\Delta G^{*}=\frac{16 \pi \sigma_{\mathrm{vn}}^{2}}{3 \Delta g_{\mathrm{v}}}\left(\frac{2-3 \cos \theta+\cos ^{3} \theta}{4}\right)= \\
\frac{16 \pi \sigma_{\mathrm{vn}}^{2}}{3 \Delta g_{\mathrm{v}}} \varphi(\theta)
\end{gathered}
$$

根据数学计算可知: $0 \leqslant \varphi(\theta) \leqslant 1$, 即非自发形 核所需要能量低于自发形核所需要的能量, 所以气 相沉积于基体后更倾向于非自发性形核机制。

此外, 非自发形核率

$$
N=\operatorname{Cexp}\left(\frac{E_{\mathrm{d}}-E_{\mathrm{s}}-\Delta G^{*}}{\mathrm{k} T}\right)
$$

其中, $C$ 是常数, $E_{\mathrm{d}}$ 和 $E_{\mathrm{s}}$ 吸附原子的脱附激活能 和扩散激活能。

非自发形核的临界半径和形核率受气相浓度和 基体温度的双重影响 ${ }^{[31-32]}$, 最终导致涂层结构的 差异 ${ }^{[18]}$ 。在本实验中当以喷距 650 1250 mm 沉积时, PS-PVD 在较高的沉积率和相对较低的基体温度作 用下, $r^{*}$ 和 $\Delta G^{*}$ 较小, 但由于基体温度偏低, 原子扩 散能力也大大降低, 导致形核率亦偏小, 最终阴影 作用下形成如图 2 所示的细锥柱状结构。

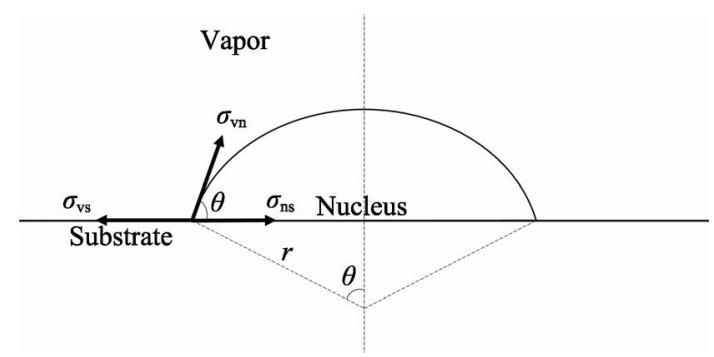

图 7 非自发形核示意图

Fig. 7 Schematic diagram of non-spontaneous nucleation

涂层柱状结构不断长大, 造成表面能持续增 加。受高表面能的影响 (等效于提高环境温度), 射 流中以自发形核的方式凝聚的晶核落于柱状结构后 有了进一步生长的可能。此时, 涂层的生长进入新 的阶段。以新核为基础发展出新的分枝, 从而形成 PS-PVD 独特的柱状结构 ${ }^{[22]}$ 。在动态平衡下, 射流 的温度和过饱和度随着喷距的增大而减小。基于上 述过饱和自发形核和过冷自发形核的讨论 (由于喷 距较短, 以前者为主), 喷距越小, 形核数量越多, 喷距越大, 形核的尺寸越大。新核沉积于柱状结构 后, 在较高浓度的气相沉积下, 有一定可能发展成 柱状结构枝晶, 临界核尺寸越大, 发展成枝晶的可 能性越高。因此, 在喷距 $650 \mathrm{~mm}$ 处, 通过自发形 核形成的晶核数量较多, 但尺寸较小, 所以发展成 枝晶的比例并不高, 且分布广泛, 所以单个花菜头 的枝晶数量并不多, 故花菜头较小, 且表面粗糙度 低; $950 \mathrm{~mm}$ 处形核尺寸较大, 形核率也不低, 分布 较为广泛, 所以单个柱状结构生长出枝晶较多, 故 花菜头较大, 表面粗䊁度较高; $1250 \mathrm{~mm}$ 处晶核尺 寸相比 $950 \mathrm{~mm}$ 处的更大, 更容易发展成枝晶, 但 数量较少, 所以单个枝晶可以吸收更多的气相和新 核, 长出更多的次级枝晶, 故喷距 $1250 \mathrm{~mm}$ 处单个 柱状结构尺寸大, 相应的“花菜头”的尺寸也大, 导 致涂层表面粗䊁度也高。

\section{3 结论}

基于不同喷距下 PS-PVD制备的 7YSZ 涂层的微 观结构和相成分研究及 OES 光谱诊断结果, 探讨了 不同轴向下 PS-PVD 7YSZ 涂层的三种沉积机制, 结 论如下:

1）喷距 $350 \mathrm{~mm}$ 处, 高浓度气相粒在基体前发生 过饱和自发形核和液/固粒子共同作用形成致密结构涂 层, 主要相成分为四方相 $\left(\mathrm{t}^{\prime}\right)$ 和单斜相 $(\mathrm{m})$ 氧化锆。

2) 喷距 650 1250 mm 范围内, 涂层具有典型的 柱状结构, 主要相成分是 t'- $\mathrm{ZrO}_{2}$ 。以非自发形核为主, 以岛状模式生长。在涂层的生长过程中, 受高表面能 
影响，允许沉积于柱状结构上的由气相自发形核形 成新核，在其基础上形成新的分支。受射流中气相浓 度和射流温度的影响, 新核的尺寸和数量存在较大 差异，造成不同喷距下形成的柱状结构也不相同。

3) 喷距 $1800 \mathrm{~mm}$ 处, 涂层亦为致密结构, 主要 由 $\mathrm{t}^{\prime}-\mathrm{ZrO}_{2}$ 构成。这是由于靠近射流尾端, 射流加热 能力不断减弱, 当粉末所处位置的环境温度低于粉 末沸点时, 粉末发生过冷自发形核, 新核随着距离 的增加而长大，最终沉积于基体上。

\section{参考文献:}

[1] REFKE A, GINDRAT M, VON NIESSEN K, et al. LPPS Thin Film: a Hybrid Coating Technology between Thermal Spray and PVD for Functional Thin Coatings and Large Area Applications. ITSC, Beijing, 2007: 14-16.

[2] DORIER J L, GINDRAT M, HOLLENSTEIN C, et al. Plasma Jet Properties in a New Spraying Process at Low Pressure for Large Area Thin Film Deposition. ITSC, Singapore, 2001: 759-764.

[3] HALL A C, MCCLOSKEY J F, URREA D A, et al. Low Pressure Plasma Spray-Thin Film ${ }^{\circledR}$ at Sandia National Laboratories. ITSC, 2009: 725-728.

[4] SMITH M F, HALL A C, FLEETWOOD J D, et al. Very low pressure plasma spray - a review of an emerging technology in the thermal spray community. Coatings, 2011, 1(2): 117-132.

[5] MAUER G, VAßEN R, STOVER D. Thin and dense ceramic coatings by plasma spraying at very low pressure. Journal of Thermal Spray Technology, 2010, 19(1/2): 495-501.

[6] SHINOZAWA A, EGUCHI K, KAMBARA M, et al. Feather-like structured YSZ coatings at fast rates by plasma spray physical vapor deposition. Journal of Thermal Spray Technology, 2010, 19(1/2): 190-197.

[7] VON NIESSEN K, GINDRAT M, REFKE A. Vapor phase deposition using plasma spray-PVD ${ }^{\mathrm{TM}}$. Journal of Thermal Spray Technology, 2010, 19(1/2): 502-509.

[8] KAMBARA M, SHINOZAWA A, AOSHIKA K, et al. Development of porous YSZ coatings with modified thermal and optical properties by plasma spray physical vapor deposition. Journal of Solid Mechanics and Materials Engineering, 2010, 4(2): 94-106.

[9] MAUER G. Plasma characteristics and plasma-feedstock interaction under PS-PVD process conditions. Plasma Chemistry and Plasma Processing, 2014, 34(5): 1171-1186.

[10] SCHMITT M P, HARDER B J, WOLFE D E. Process-structureproperty relations for the erosion durability of plasma spray- physical vapor deposition (PS-PVD) thermal barrier coatings. Surface and Coatings Technology, 2016, 297: 11-18.

[11] REFKE A, HAWLEY D, DOESBURG J, et al. LPPS Thin Film Technology for the Application of TBC Systems. ITSC, Basel, 2005: 438-443

[12] HOSPACH A, MAUER G, VAßEN R, et al. Columnar-structured thermal barrier coatings (TBCs) by thin film low-pressure plasma spraying (LPPS-TF). Journal of Thermal Spray Technology, 2011, 20(1/2): 116-120.

[13] GORAL M, KOTOWSKI S, NOWOTNIK A, et al. PS-PVD deposition of thermal barrier coatings. Surface and Coatings Technology, 2013, 237: 51-55.

[14] REZANKA S, MAUER G, VAßEN R. Improved thermal cycling durability of thermal barrier coatings manufactured by PS-PVD. Journal of Thermal Spray Technology, 2014, 23(1/2): 182-189.

[15] SONG J, ZHANG X, DENG C, et al. Research of in situ modified PS-PVD thermal barrier coating against CMAS (CaO-MgO- $\mathrm{A}_{12}$ $\mathrm{O}_{3}-\mathrm{SiO}_{2}$ ) corrosion. Ceramics International, 2016, 42(2): 31633169.

[16] GAO L, GUO H, WEI L, et al. Microstructure, thermal conductivity and thermal cycling behavior of thermal barrier coatings prepared by plasma spray physical vapor deposition. Surface and Coatings Technology, 2015, 276: 424-430.

[17] MAUER G, HOSPACH A, VAßEN R. Process development and coating characteristics of plasma spray-PVD. Surface and Coatings Technology, 2013, 220: 219-224.

[18] MAUER G, HOSPACH A, ZOTOV N, et al. Process conditions and microstructures of ceramic coatings by gas phase deposition based on plasma spraying. Journal of Thermal Spray Technology, 2013, 22(2/3): 83-89.

[19] VON NIESSEN K, GINDRAT M. Plasma spray-PVD: a new thermal spray process to deposit out of the vapor phase. Journal of Thermal Spray Technology, 2011, 20(4): 736-743.

[20] MAUER G, VAßEN R. Plasma spray-PVD: plasma characteristics and impact on coating properties. Journal of Physics: Conference Series, 2012, 406: 012005-012017.

[21] LI C, GUO H, GAO L, et al. Microstructures of yttria-stabilized zirconia coatings by plasma spray-physical vapor deposition. Journal of Thermal Spray Technology, 2015, 24(3): 534-541.

[22] GAO L, WEI L, GUO H, et al. Deposition mechanisms of yttria-stabilized zirconia coatings during plasma spray physical vapor deposition. Ceramics International, 2016, 42(4): 5530-5536.

[23] GAO L, GUO H, WEI L, et al. Microstructure and mechanical properties of yttria stabilized zirconia coatings prepared by plasma spray physical vapor deposition. Ceramics International, 2015, 41(7): 8305-8311.

[24] ZHANG X F, ZHOU K S, DENG C M, et al. Gas-deposition mechanisms of 7YSZ coating based on plasma spray-physical vapor deposition. Journal of the European Ceramic Society, 2016, 36(3): 697-703.

[25] ZHANG X F, ZHOU K S, SONG J B, et al. Deposition and CMAS corrosion mechanism of 7YSZ thermal barrier coatings prepared by plasma spray-physical vapor deposition. Journal of Inorganic Materials, 2015, 30(3): 287-293.

[26] GINDRAT M. Characterization of Supersonic Low Pressure Plasma Jets. Doctoral Thesis, 2004.

[27] ZHANG X F, ZHOU K S, ZHANG J F, et al. Structure evolution of 7YSZ thermal barrier coating during thermal shock testing. Journal of Inorganic Materials, 2015, 30(12): 1261-1266.

[28] GARVIE R C, HANNINK R H, PASCOE R T. Ceramic steel? Nature, 1975, 258(5537): 703-704.

[29] HAN P, YOSHIDA T. Numerical investigation of thermophoretic effects on cluster transport during thermal plasma deposition process. Journal of applied physics, 2002, 91(4): 1814-1818.

[30] ORHING M. Materials Science of Thin Films: Deposition and Structure, 2nd ed. London: Academic Press, 2001: 357-406.

[31] VENABLES J A, SPILLER G D T, HANBUCKEN M. Nucleation and growth of thin films. Reports on Progress in Physics, 1984, 47(4): 399.

[32] THORNTON J A. Influence of substrate temperature and deposition rate on structure of thick sputtered Cu coatings. Journal of Vacuum Science and Technology, 1975, 12(4): 830-835. 